\title{
Visual space: a test of the constant curvature hypothesis
}

\author{
John M. Foley \\ COLUMBIA UNIVERSITY ${ }^{1}$
}

\begin{abstract}
The Desarguesian property, an implication of the constant curvature hypothesis was submitted to experimental test. This property requires that, if two vertices of a triangle are joined by segments to the opposite sides, these segments intersect. The results indicate that the property does hold for a significant portion of observers; others may be slightly non-Desarguesian.
\end{abstract}

\section{Problem}

Visual space is the space determined by a set of visible points, and the apparant relations among them, viewed binocularly. The problem investigated here concerns the possibility of representing this space by a geometrical space. Luneburg (1947) proposed that it might be represented by a Riemannian space of constant negative curvature, a hyperbolic space. In a recent reformulation of the theory Blank (1958) indicates that a necessary condition for constant curvature is that the space be Desarguesian, i.e. if two vertices of a triangle are joined by segments to the opposite sides, these segments intersect (Fig. 1). This study is a test of the Desarguesian property.

\section{Method}

Three-dimensional configurations of small, pointlike, lights were produced by the polaroid stereoscopic technique. The stimulus for each light was a laterally separated pair of lights. One light of each pair was visible to each eye, resulting in the impression of a single light. The distance of these simulated lights was controlled by varying the lateral separation of the pair; their left-right position, by moving the pair along a horizontal bar. The lights were viewed binocularly with the head fixed and the eyes allowed to move freely.

The observer was presented with three lights $\mathrm{P}_{\mathrm{a}}$, $\mathrm{P}_{\mathrm{b}}$, and $\mathrm{P}_{\mathrm{c}}$ forming a fixed triangle (Fig. 1). A fourth light $Q_{\cdot a}$ was introduced. This light was moved by the experimenter according to instructions signalled by the observer until it appeared to be aligned between vertices $\mathrm{P}_{\mathrm{b}}$ and $\mathrm{P}_{\mathrm{c}}$. (The position of the horizontal bar determined the plane in which each light moved and thus the spacing of the lights on the lines.) Four additional lights were then introduced, which the observer aligned between $\mathrm{Q}_{\mathrm{a}}$ and $\mathrm{P}_{\mathrm{a}}$. All lights except the fixed triangle were then extinguished and the procedure was repeated, first aligning $Q_{c}$ between $P_{a}$ and $P_{b}$, then using the other four variable lights to construct the segment be-

${ }^{1}$ Now at the University of California, Santa Barbara tween $Q_{\epsilon}$ and $P_{C}$. Each of the two segments was constructed 24 times in counterbalanced order. Four segments were constructed at each experimental session.

Two triangles were chosen by a random process with constraints insuring that they be large. One, T1, extended $20^{\circ}$ from the median plane into the periphery; the other, T2, extended 16.6 on both sides of the median plane. The first had a greater depth dimension. Five observers participated in the study. For each triangle two observers constructed segments on that triangle alone. The remaining observer constructed segments on both triangles.

For each setting of the two segments the length of shortest join between them was determined using vector methods. Average values were determined for each component of the shortest join. Statistical tests were performed to determine if the mean lengths of these components were significantly different from zero.

\section{Results}

In general the locus of points corresponding to an apparently straight segment was found to be a curve slightly concave toward the observer. Table 1 gives the mean, standard deviation, and $95 \%$

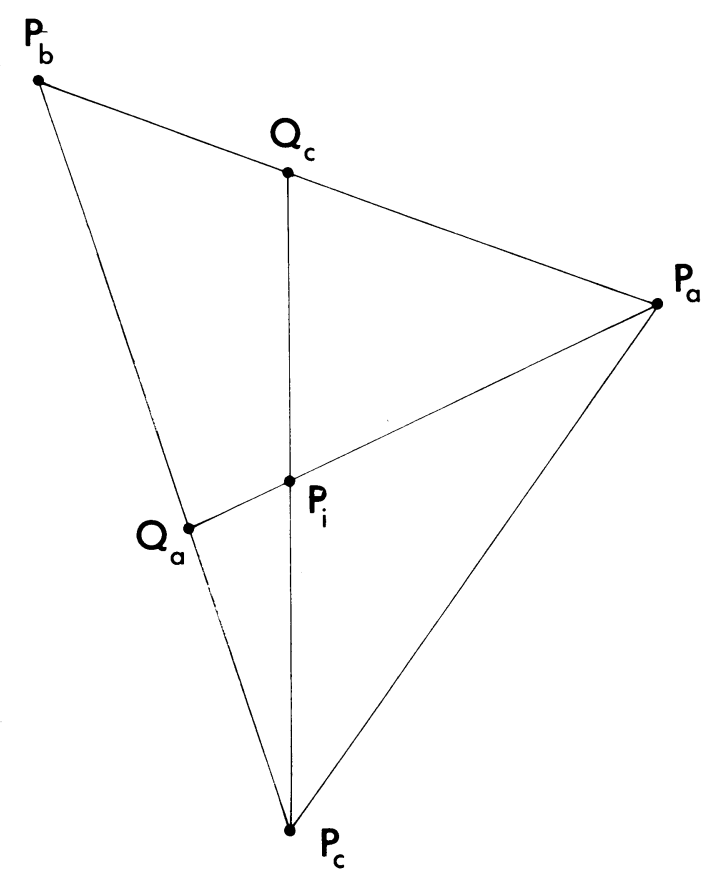

Fig. 1. Illustration of the Desarguesian property. The property requires that $P_{a} Q_{a}$ and $P_{c} Q_{c}$ intersect. It is a necessary condition for constant curvature. 
TABLE 1. Mean,* Standard Deviation, and 95\% Confidence Limits for Components of the Shortest Join in Simulated Coordinates (All values are in $\mathrm{cm} ; \mathrm{N}=24$ )

\begin{tabular}{|c|c|c|c|c|c|c|}
\hline \multirow[b]{2}{*}{ Observer } & \multicolumn{3}{|c|}{ Triangle 1} & \multicolumn{3}{|c|}{ Triangle 2} \\
\hline & 2 & 3 & 4 & 2 & 5 & 6 \\
\hline Mean, $\mathrm{X}$ coordinate & .84 & .97 & 2.09 & -.74 & 1.12 & 15.69 \\
\hline Standard deviation & .99 & 1.00 & 1.59 & 2.68 & 8.70 & 25.69 \\
\hline Lower bound** & .42 & .55 & 1.42 & -1.87 & -2.55 & -26.54 \\
\hline Upper bound** & 1.26 & 1.39 & 2.76 & .09 & 4.79 & -4.84 \\
\hline Mean, Y coordinate & -2.28 & -3.43 & -5.27 & .81 & .60 & 5.86 \\
\hline Standard deviation & 2.30 & 3.33 & 3.92 & 3.21 & 6.25 & 11.76 \\
\hline Lower bound** & -3.25 & -4.83 & -6.92 & -.54 & -2.04 & .89 \\
\hline Upper bound** & -1.31 & -2.03 & -3.62 & 4.02 & 3.24 & 10.83 \\
\hline Mean, $\mathrm{Z}$ coordinate & -1.15 & -1.65 & -2.79 & -.48 & .36 & -6.37 \\
\hline Standard deviation & 1.37 & 1.70 & 2.15 & 1.87 & 7.47 & 9.54 \\
\hline Lower bound $* *$ & -1.73 & -2.36 & -3.69 & -1.27 & -2.80 & -2.34 \\
\hline Upper bound** & -.57 & -.94 & -1.89 & .31 & 3.52 & -10.40 \\
\hline
\end{tabular}

* Positive values indicate that point of shortest join on line A lies in front, to the left, or above the point of the shortest join in line $\mathrm{C}$.

** of $95 \%$ confidence interval

confidence limits for each component of the shortest join. In all cases the length of the join is short relative to the size of the triangles and to the variability of the settings. In terms of visual angle the lengths of the shortest join range from 20 to $220 \mathrm{sec}$. In two cases, Observers 2 and 5 on Triangle 2 , the confidence intervals contain zero for each component indicating that the length of the shortest join is not significantly different from zero at the .05 level of significance. In the other cases the length is significantly different from zero.

Since the Desarguesian property refers to the visual space, it does not require that the segments intersect physically, but only that they appear to intersect. Consequently a supplementary experiment was carried out to determine the threshold for the discrimination of the non-intersection of two such segments. Observers 2 and 4 participated in this study. Four lights were presented to the observer. He varied the distance of one of them until the segment determined by one pair appeared to intersect the segment determined by the other pair. Under conditions similar to those of the main experiment, threshold values were found to lie in the range of 59 to $92 \mathrm{sec}$.

\section{Conclusions}

The Desarguesian property may be considered to hold in the two cases where the length of the shortest join was found to be not significantly different from zero. In the other cases, a decision is more difficult. The results of the supplementary experiment suggest that in at least some of the cases where the join length is significantly different from zero, it is still below the threshold of discrimination. Other cases may be slightly non-Desarguesian. If they are, the deviations are small relative to the size of the configurations and may not be large enough to interfere with the usefulness of the theory.

The results suggest that the length of the shortest join may depend on the extent to which the triangle extends into the periphery of the visual field. Three of the four cases in which the shortest join was significantly long occurred in the triangle extended farthest from the median plane, Triangle 1.

\section{heferences}

BLANK, A. A. Axiomatics of binocular vision. The foundations of metric geometry in relation to space perception. J. Opt. Soc. Amer.,1958a, 48, 328-334.

BLANK, A. A. Analysis of experiments in binocular space perception. J. Opt. Soc. Amer., 1958b, 48, 911925 .

LUNEBURG, R. K. Mathematical analysis of binocular vision. Princeton: Princeton University Press, 1947.

\section{Acknowledgment}

This research was supported in part by a contract between the U. S. Office of Naval Research and Columbia University. The author is grateful to Professor Clarence H. Graham and Professor Albert A. Blank, who served as advisors on this project. 